OPEN ACCESS

Edited by:

Yonghan $\mathrm{He}$

Chinese Academy of Sciences, China

Reviewed by:

Carsten Merkwirth

Ferring Research Institute, Inc.

United States

lonel Sandovici,

University of Cambridge,

United Kingdom

Fu-Hui Xiao,

Kunming Institute of Zoology (CAS),

China

*Correspondence:

Alexander Vaiserman

vaiserman@geront.kiev.ua;

vaiserman23@gmail.com

Specialty section

This article was submitted to

Genetics of Aging,

a section of the journal

Frontiers in Genetics

Received: 01 October 2018 Accepted: 09 November 2018 Published: 22 November 2018

Citation:

Vaiserman A (2018)

Developmental Tuning of Epigenetic Clock. Front. Genet. 9:584.

doi: 10.3389/fgene.2018.00584

\section{Developmental Tuning of Epigenetic Clock}

\author{
Alexander Vaiserman* \\ Institute of Gerontology, Kiev, Ukraine
}

Research in the field of gerontology has traditionally focused on later life stages. There is increasing evidence, however, that both the rate of age-related functional decline and the later-life health status can be programmed during early development. The central role of epigenetic mechanisms (methylation of DNA, histone modifications and regulation by non-coding RNAs) in mediating these long-term effects has been elucidated. Both rate and direction of age-associated change of epigenetic patterns ("epigenetic drift") were shown to be largely dependent on early-life environmental conditions. Inter-individual divergences in epigenetic profiles may arise following the stochastic errors in maintaining epigenetic marks, but they may also be adaptively mediated by specific environmental cues. Recent cohort studies indicate that ticking rate of epigenetic clock, estimated by a DNA methylation-based methods, may be developmentally adjusted, and that individual's discrepancies among epigenetic and chronological age would be likely programmed early in development. In this Perspective article, recent findings suggesting the importance of early-life determinants for life-course dynamics of epigenetic drift are summarized and discussed.

Keywords: DNA methylation, age-related disease, aging rate, developmental programming, epigenetic clock, epigenetic drift

\section{INTRODUCTION}

Genetic and lifestyle factors have been traditionally considered as main determinants of aging rate and longevity. However, accumulating data indicate that both individual's aging trajectory and population mortality rate may substantially depend on developmental conditions (TarryAdkins and Ozanne, 2014; Vaiserman et al., 2018). The mechanistic pathways underlying such life-long effects are largely unknown, but modulation of epigenetic regulation of gene expression appears to be the most plausible explanation (Bianco-Miotto et al., 2017). Epigenetic modifications refer to heritable changes in gene expression occurring without changes in DNA sequence. Main components of epigenetic control include DNA methylation, histone modifications, and regulation by non-coding RNAs. Initially, it was assumed that stochastic errors in maintaining epigenetic marks ("epimutations") occur during the life course due to limitations in epigenetic settings of maintenance and repair functions (Holliday, 1987). Since age-related accumulation of epimutations inevitably leads to impairment of the normal gene responsiveness to environmental stresses and to gradual loss of phenotypic plasticity, this process is commonly believed to be one of the main causes of aging (Gravina and Vijg, 2010). In addition, since age-related loss of DNA methylation may result in chromosome instability, and hypermethylation of DNA in promoter regions of tumor suppressor genes can cause their suppression, accumulation of epimutations with age could play an important role in the initiation and progression of different cancers (Jung and Pfeifer, 2015; Feinberg et al., 2016). 
An accumulation of mistakes in maintaining normal epigenetic patterns leads to an increase in variability of epigenetic marks throughout the individual's life course. This process currently referred to as "epigenetic drift" results in a gradual impairment of the body's homeostatic mechanisms and appears to be a crucial hallmark of aging and an important determinant of longevity (Mendelsohn and Larrick, 2017). The earliest evidence for epigenetic drift in humans was provided from studies of monozygotic (MZ) twins. Withinpair comparison of such twins provides a useful model for investigation of factors regulating epigenetic variability by controlling for genetic variation. Despite MZ twins have the same genetic background their epigenetic profiles have been shown to gradually diverge with age (Fraga et al., 2005). Remarkably, most apparent divergences in epigenetic profiles have been observed in MZ twins who, according to questionnaire responses, had spent less of their lifetime together and/or had more differing natural health-medical history. Similarly, in recent study by Li et al. (2018), the correlation in genome-wide average DNA methylation levels between non-twin first-degree relatives was shown to increase with time living together and decrease with time living apart. An interaction between environmental conditions and age-related methylation divergence was also confirmed in a longitudinal, genome-scale analysis of DNA methylation in $\mathrm{MZ}$ twins from birth to 18 months (Martino et al., 2013). These findings collectively suggest that divergence in epigenetic profiles can occur not only via the stochastic epigenetic drift caused, e.g., by errors in the maintenance of DNA methylation patterns throughout DNA replication cycles, but may also be mediated directly by specific environmental cues (Cortessis et al., 2012; Cunliffe, 2015). From this, it is assumed that epigenome can respond to environmental challenges in an adaptive manner to maintain homeostasis and performance (Vaiserman, 2008, 2010; Herman et al., 2014). Thereby, the age-associated epigenome modification is increasingly seen as a process of memorization of environmental exposures experienced by organism over the lifetime. The epigenetic memorization likely affects the epigenetic drift rate. In this Perspective article, recent findings suggesting the importance of early-life determinants for life-course dynamics of epigenetic drift are summarized and discussed.

\section{EARLY-LIFE DETERMINANTS OF EPIGENETIC DRIFT}

Multiple lines of evidence indicate that both direction and rate of age-associated epigenetic drift largely depend on earlylife environmental conditions ( $\mathrm{Li}$ and Tollefsbol, 2016). This is not surprising, since it is well known that the epigenome (the whole-genome totality of epigenetic marks) is highly plastic during the early developmental period, especially throughout the establishment of the cell lineage-specific profiles of gene expression (Iurlaro et al., 2017; Burns et al., 2018). In mammals, including humans, windows of early-life epigenetic plasticity extend from preconception through weaning (Vaiserman, 2015). After the establishment throughout early developmental stages, most epigenetic marks are stably propagated in course of numerous cell divisions. Such epigenetic cellular memory allows to maintain stable profiles of gene expression in particular cell lineages throughout lifetime. This process is commonly referred to as "developmental epigenetic programming" (Hochberg et al., 2011). Importance of this period to future health status underlies the concept of the "first 1000 days," prioritizing gestation and first 2 years after birth as a critical developmental period (Garmendia et al., 2014). According to the concept of predictive adaptive response, early-life cues can be used by an organism to rearrangement of the epigenome in a way that provides greatest fitness dividends in future life (Bateson et al., 2014). If resulting phenotype is properly matched to predicted environmental conditions, such adaptive strategy results in survival benefits in adult life. If adaptive epigenetic fine-tuning in early life is, however, incorrect and a mismatch exists between the further living conditions and the developmentally programmed phenotype, it may cause increased disease risk in adulthood (Godfrey et al., 2007). These conceptual considerations seem particularly relevant in the context of the topic discussed. The research findings suggesting the importance of early-life determinants for epigenetic programming of aging phenotypes and dynamics of epigenetic drift are presented in subsequent sections.

\section{Evidence From Animal Models}

Since human data are scarce owing to restricted access to suitable biological materials, the most direct evidence for the role of epigenetic regulation in developmental programming of aging and longevity phenotypes came from animal models. In Merkwirth et al. (2016) study, perturbation of mitochondria throughout larval development not only delayed aging but also maintained unfolded protein response [UPR $(\mathrm{mt})]$ signaling in Caenorhabditis elegans, assuming an epigenetic mechanism contributing to both lifespan and mitochondrial proteostasis across life course. In particular, reducing the function of lysine demethylases, JMJD-1.2/PHF8 and JMJD-3.1/JMJD3, has been shown to be able to potentially suppress longevity and UPR(mt) induction, while gain of function was sufficient to extend lifespan in a UPR(mt)-dependent manner. In the Greer et al. (2010) study, the ASH-2 trithorax complex, which trimethylates histone $\mathrm{H} 3$ at lysine 4 (H3K4), was identified as another important player in epigenetic programming of longevity in nematode. Remarkably, developmental activation of a particular longevity-regulating signaling pathways caused by misregulation of $\mathrm{H} 3 \mathrm{~K} 4 \mathrm{me} 2$, led to a transgenerational lifespan extension in F2-F4 nematode offspring, potentially indicative of epigenetic memory (Greer et al., 2011). The possibility of such epigenetically mediated transgenerational effects on the worm's longevity has been also confirmed in more recent studies (Alvares et al., 2014; Greer et al., 2016; Kishimoto et al., 2017).

Transgenerational effects on reproductive activity and longevity of Drosophila melanogaster induced by post-eclosion manipulation with dietary protein content were reported by Xia and de Belle (2016). In this research, both low- and highprotein diets reduced lifespan, while the intermediate-protein diet significantly extended longevity until F3 generation. In a 
subsequent study, feeding with a low-protein diet throughout the post-eclosion period resulted in a shortened life expectancy in F0 generation as well as in F2 offspring. These effects were accompanied by upregulating the H3K27-specific methyltransferase, $\mathrm{E}(\mathrm{z})$, and enhanced levels of H3K27 trimethylation, H3K27me3 (Xia et al., 2016). Interestingly, both RNAi-mediated knockdown of $\mathrm{E}(\mathrm{z})$ and pharmacological inhibiting its enzymatic function with a specific inhibitor of histone methyltransferase, Tazemetostat (EPZ-6438), lowered H3K27me3 levels across generations. In addition, Tazemetostat completely abolished the lifespan-shortening effect of the parental low-protein diet.

In rodent models, confirmatory evidence was also obtained for the role of factors such as xenobiotic exposure, stress, and malnutrition in developmental epigenetic programming of pathways contributed to the control of aging and longevity (for reviews, see Vaiserman, 2014; Tarry-Adkins and Ozanne, 2017; Ambeskovic et al., 2018). For example, in the study by Heo et al. (2016), prenatal malnutrition led to disrupted patterns of DNA methylation and dysregulation of transcriptional activity of genes implicated in aging-related processes and development of metabolic disorders in young (9-week-old) rat offspring. Remarkably, these dysregulated epigenetic patterns have been similar to those revealed in aged (20-month-old) offspring.

\section{Evidence From Twin Models}

The evidence that epigenetic drift can be developmentally programmed was mostly obtained from studies in birth-weight discordant MZ twins. Birth weight was used in these studies as a proxy for prenatal conditions. Such research design generally relies on investigating twins raised in shared family environments, which may provide control not only for common genetic background but also for similar postnatal rearing environment. In the study by Gordon et al. (2011), strong evidence of gene expression discordance in MZ twins at birth was found in two different cell types. Among genes showing most different expression patterns within pairs, there were genes involved in stress response, metabolism, and cardiovascular function. In several recent studies, evidence was also obtained that early-life-induced epigenetic alterations may persist into adulthood. Although very similar genome-wide profiles of DNA methylation were found in saliva samples from discordant for birth weight adult female MZ twins (Souren et al., 2013), more recent studies revealed persistent differences in DNA methylation profiles. Importantly, most of these differentially methylated genes were known to be implicated in aging-associated processes. Pronounced differences in fetal growth (discordance in birth weight more than 20\%) were significantly associated with DNA methylation changes in the IGF1R gene during adulthood (Tsai et al., 2015). In an epigenome-wide DNA methylation analysis conducted to examine adult MZ twins discordant for birth weight, a region on chromosome 1 has been identified as being differentially methylated for birth-weight discordance (Chen et al., 2016). This region covered two metabolism-associated genes, CRYZ and TYW3. Genome-wide methylome profiling in blood samples from adult MZ twins discordant for birth weight did not reveal any differences in DNA methylation patterns between twins, although particular sites displayed ageassociated intra-pair differential methylation in highly birth weight-discordant twin pairs (Tan et al., 2014). The evidence that life-course dynamics of epigenetic drift is largely dependent from early-life programming events was also obtained from nonMZ twin models. In analyzing results from seven twin and/or family studies, Li et al. (2018) found that correlation in genomewide average DNA methylation levels is very high at birth and remains high enough throughout life course in both twins and non-twin first-degree relatives. On the basis of these data, the authors concluded that genome-wide DNA methylation levels are determined in utero by prenatal environmental exposures, and these effects persist throughout life.

\section{Quasi-Experimental Evidence}

Research findings indicative of contribution of epigenetic mechanisms to developmental programming of adverse laterlife health outcomes were also obtained in quasi-experimental studies. Such research design (also referred to as "natural experiment") is defined as "naturally occurring circumstances in which subsets of the population have different levels of exposure to a supposed causal factor, in a situation resembling an actual experiment where human subjects would be randomly allocated to groups" (Last, 1995). The Dutch famine, that took place in the German-occupied part of Netherlands in 19441945 , is the most studied in this respect. The signs of accelerated aging were repeatedly reported in the cohort born during this famine, including impaired physical function, lowered cognitive performance, more atherogenic plasma lipid profile, enhanced stress responsivity, increased risk for depression and cardiometabolic disorders (Lumey et al., 2011; Roseboom et al., 2011; Bleker et al., 2016), and also the enhanced mortality rate (van Abeelen et al., 2012) at older ages. These adverse health outcomes were accompanied by persistent epigenetic changes. Whereas no association has been reported between prenatal exposure to the Dutch famine and the overall level of DNA methylation in adult life (Lumey et al., 2012), levels of DNA methylation of particular genes have been strongly associated with prenatal exposure to famine in the whole blood samples from adult offspring. Among the genes found to be differentially methylated between the famine-exposed subjects and non-exposed control individuals, there were genes known to be implicated in the development of cardiometabolic phenotypes, such as IGF2 (Heijmans et al., 2008), and also GNASAS, IL10, LEP, ABCA1, INSIGF, and MEG3 (Tobi et al., 2009). More recently, differential methylation of genomic regions extended along pathways associated with growth and metabolism was observed in persons periconceptionally exposed to famine (Tobi et al., 2014). Early, but not mid or late gestation, was identified as a critical time window for inducing persistent changes in DNA methylation profiles (Tobi et al., 2015). Even though it has not been reported until now whether it is a correlation between the DNA methylation and gene expression levels, the revealed DNA methylation modification was clearly related to impaired metabolic homeostasis in adult individuals who were prenatally exposed to famine (Lumey et al., 2007; Tobi et al., 2018). Similar results were obtained in studying long-term consequences of the 

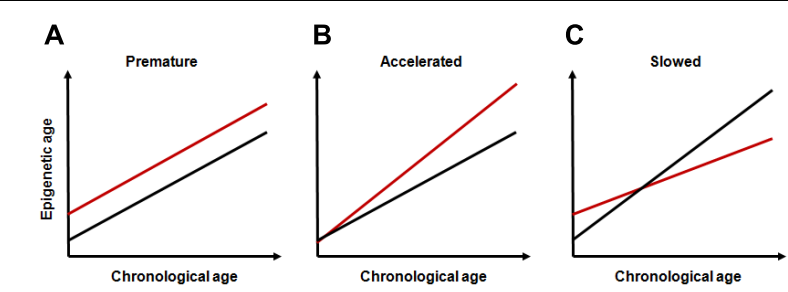

FIGURE 1 | Hypothetical modes of life-course dynamics of epigenetic aging (A) premature epigenetic aging; (B) accelerated epigenetic aging; and (C) slowed epigenetic aging. In all panels, red lines represent subjects exposed to adverse early-life events and black lines represent unexposed subjects.

perinatal exposure to famine in rural Bangladesh. In this study, those offspring who were exposed to famine perinatally were demonstrated to be at higher risk of developing obesity and type 2 diabetes during adulthood in comparison with unexposed control subjects. These health outcomes were associated with significant differences in methylation levels in metastable epialleles such as PRDM-9, VTRNA2-1, PAX8, near BOLA, near ZFP57, and EXD3 (Finer et al., 2016).

\section{DEVELOPMENTAL ADJUSTMENT OF EPIGENETIC CLOCK}

Recently, DNA methylation has gained significant interest as a powerful biomarker of aging allowing to quantify the individual aging rate and inter-individual variations in functional decline and timing of disease onset during the life course (Levine et al., 2018). In particular, the multi-tissue algorithm developed by Horvath (2013) allows to produce age estimates which correlate with chronological age well above $r=0.90$ for full age range samples. Recent cohort studies indicated that ticking rate of epigenetic clock, estimated by DNA methylation-based methods, may be developmentally adjusted, and that individual's discrepancies between epigenetic and chronological age may be programmed early in life. The supporting evidence for this came mainly from cohort studies. In most of these studies, the differences between actual chronological age and calculated DNA methylation age have been estimated using Horvath's method (Horvath, 2013). For example, dependence of adult rate of epigenetic aging (age acceleration, AgeAccel) from childhood conditions was evaluated in the study by Simpkin et al. (2016). AgeAccel was defined as residual from regressing epigenetic age on actual age. The association between birth weight and AgeAccel during adolescence was found in two birth cohorts in this study. In analysis of the profiles of DNA methylation across five timepoints in mother-child pairs from a United Kingdom birth cohort, adolescent AgeAccel was associated with maternal alcohol consumption during pregnancy. In a subsequent longitudinal study conducted with the same birth cohort, strong associations between AgeAccel and several developmental characteristics including height, weight, fat mass, and body mass index during childhood and adolescence have been demonstrated (Simpkin et al., 2017). An inverse association between pubertal tempo and
AgeAccel in girls was shown in the longitudinal Growth and Obesity Cohort Study (Binder et al., 2018). Similar findings were obtained in two Finnish follow-up cohorts where AgeAccel levels were established in pre-adult life and remained unchanged during the rest of the lifetime, even in oldest-old ages (Kananen et al., 2016). On the basis of these findings, the epigenetic clock theory of aging was recently proposed by Horvath and Raj (2018), which postulated that epigenetic clock links developmental processes to aging.

\section{HYPOTHETICAL CONSIDERATIONS}

Based on the conceptual frameworks and research findings above, it can be assumed that life-course dynamics of epigenetic aging can depend on early-life events and that the mode of this dependence can be different depending on the stage affected and on the type, duration and intensity of exposure. Most data on this relationship correspond to common intuitive notions. Adverse environmental exposures early in life can result in an elevated rate of accumulation of epimutations without accelerating the rate of epigenetic aging. This may increase intercept parameter without changing the slope parameter in the linear regression model describing this relationship ("premature epigenetic aging," Figure 1A). Inappropriate developmental programming due to, e.g., mismatched epigenetic adaptation, may cause increase in slope ("accelerated epigenetic aging," Figure 1B).

Some findings on long-term consequences of early-life programming events are, however, counterintuitive and require additional assumptions. For example, in analyzing the deceleration of the old-age mortality rate observed across developed countries over the second part of the XXth century, Yashin et al. (2001) suggested that some centenarians paradoxically come from an initially frail part of the cohort. The authors hypothesized that more vulnerable (and likely more labile) organism can better improve its stress reactivity than more robust (and rigid) one. This can result in survival advantages to originally more vulnerable organisms at older ages. The evidence for this assumption also comes from studies conducted to test hygiene hypothesis proposed by Strachan (1989) to explain the link between the lack of microbial exposure due to overhygienic conditions in childhood and high risk of allergic and autoimmune disorders in later life. The overhygienization is becoming increasingly widespread in modern world, and it is believed to cause increased antibiotic resistance and permanently increasing number of persons with weakened immunity in developed societies (Bloomfield et al., 2016). Although lack of microbial exposure may not be the only causal factor, the assumptions of this hypothesis have been repeatedly confirmed through both observational and experimental studies (Maia Rda and Wünsch Filho, 2013). Under-using of immune system early in life can likely cause not only atopic conditions, but also autoimmune states (Kramer et al., 2013). The latter seems especially important since immunosenescence are regarded now as one of the leading processes underlying aging. Importantly, although inflamm-aging is traditionally regarded as leading cause of most age-related disorders, such strictly negative 
interpretation of immunosenescence is challenged now by many immune-gerontologists. Age-associated immune changes are increasingly considered as adaptive or remodeling rather than solely detrimental (Fulop et al., 2018). Despite the fact that these immune changes can obviously cause various pathological conditions, these alterations may potentially contribute to developing extended longevity phenotypes. It is even suggested by the authors that without the presence of the immunosenescence/inflamm-aging, human longevity would be substantially shortened (Fulop et al., 2018). An important point in the context of the topic discussed is that complex interactions between immune and epigenetic pathways likely play an important role in these effects (Grolleau-Julius et al., 2010; Jasiulionis, 2018). Given the fact that these processes can have a significant impact on epigenome, they may likely decelerate the epigenetic clock-ticking rate by increasing the intercept parameter and by decreasing slope value ("slowed epigenetic aging," Figure 1C), even though initial level of accumulation of epimutations could be higher in early-life exposed population than in unexposed one. Interestingly, in the study by Marioni et al. (2018), epigenetic age was shown to be increasing at a slower rate than chronological age across life course, especially in the oldest population. The selection bias in which healthier individuals are more likely to reach older ages was proposed to explain these results by the authors. The individual induction due to epigenetic adaptation might, however, be an alternative explanation. For a definitive conclusion, nevertheless, more thorough research is required.

\section{CONCLUSIVE REMARKS}

Research in the field of gerontology has traditionally focused on later stages of the life cycle. There is, however, increasing evidence that the rate of age-related functional decline and

\section{REFERENCES}

Alvares, S. M., Mayberry, G. A., Joyner, E. Y., Lakowski, B., and Ahmed, S. (2014). H3K4 demethylase activities repress proliferative and postmitotic aging. Aging Cell 13, 245-253. doi: 10.1111/acel.12166

Ambeskovic, M., Roseboom, T. J., and Metz, G. A. S. (2018). Transgenerational effects of early environmental insults on aging and disease incidence. Neurosci. Biobehav. Rev. doi: 10.1016/j.neubiorev.2017.08.002 [Epub ahead of print].

Bateson, P., Gluckman, P., and Hanson, M. (2014). The biology of developmental plasticity and the predictive adaptive response hypothesis. J. Physiol. 592, 2357-2368. doi: 10.1113/jphysiol.2014.271460

Bianco-Miotto, T., Craig, J. M., Gasser, Y. P., van Dijk, S. J., and Ozanne, S. E. (2017). Epigenetics and DOHaD: from basics to birth and beyond. J. Dev. Orig. Health Dis. 11, 1-7. doi: 10.1017/S2040174417000733

Binder, A. M., Corvalan, C., Mericq, V., Pereira, A., Santos, J. L., Horvath, S., et al. (2018). Faster ticking rate of the epigenetic clock is associated with faster pubertal development in girls. Epigenetics 13, 85-94. doi: 10.1080/15592294. 2017.1414127

Bleker, L. S., de Rooij, S. R., Painter, R. C., van der Velde, N., and Roseboom, T. J. (2016). Prenatal undernutrition and physical function and frailty at the age of 68 years: the Dutch Famine Birth Cohort Study. J. Gerontol. A. Biol. Sci. Med. Sci. 71, 1306-1314. doi: 10.1093/gerona/glw081

Bloomfield, S. F., Rook, G. A., Scott, E. A., Shanahan, F., Stanwell-Smith, R., and Turner, P. (2016). Time to abandon the hygiene hypothesis: new perspectives risk for aging-associated diseases can largely depend on developmental conditions. Growing awareness of the of developmental programming in the pathogenesis of adult-life chronic pathological conditions and unraveling the mechanisms involved led to rising interest in research in this area. The developmental programming processes would likely be of particular importance in modern developed societies due to significant lifestyle changes (westernized diet, sedentary behavior, etc.), which can often conflict with adaptive epigenetic strategies programmed during development.

Two important points follow from these considerations for future research and practical applications. First, further development of epigenetic methodology will provide an opportunity to identify individuals at risk for developing certain age-related pathological condition due to earlylife malprogramming long before the clinical manifestation of the disease. Second, since epimutations, unlike genetic mutations, are potentially reversible, they may be corrected by specific nutritional and/or pharmacological interventions. The implementation of such epigenome-targeted interventions would allow to influence life-course dynamics of epigenetic age and slow down the ticking rate of the epigenetic clock to delay or decelerate the aging-related processes.

\section{AUTHOR CONTRIBUTIONS}

The author confirms being the sole contributor of this work and has approved it for publication.

\section{ACKNOWLEDGMENTS}

The author would like to thank Oksana Zabuga for the technical assistance in preparing this manuscript.

on allergic disease, the human microbiome, infectious disease prevention and the role of targeted hygiene. Perspect. Public Health 136, 213-224. doi: 10.1177/ 1757913916650225

Burns, S. B., Szyszkowicz, J. K., Luheshi, G. N., Lutz, P. E., and Turecki, G. (2018). Plasticity of the epigenome during early-life stress. Semin. Cell. Dev. Biol. 77, 115-132. doi: 10.1016/j.semcdb.2017.09.033

Chen, M., Baumbach, J., Vandin, F., Röttger, R., Barbosa, E., Dong, M., et al. (2016). Differentially methylated genomic regions in birth-weight discordant twin pairs. Ann. Hum. Genet. 80, 81-87. doi: 10.1111/ahg.12146

Cortessis, V. K., Thomas, D. C., Levine, A. J., Breton, C. V., Mack, T. M., Siegmund, K. D., et al. (2012). Environmental epigenetics: prospects for studying epigenetic mediation of exposure-response relationships. Hum. Genet. 131, 1565-1589. doi: 10.1007/s00439-012-1189-8

Cunliffe, V. T. (2015). Experience-sensitive epigenetic mechanisms, developmental plasticity, and the biological embedding of chronic disease risk. Wiley. Interdiscip. Rev. Syst. Biol. Med. 7, 53-71. doi: 10.1002/wsbm.1291

Feinberg, A. P., Koldobskiy, M. A., and Göndör, A. (2016). Epigenetic modulators, modifiers and mediators in cancer aetiology and progression. Nat. Rev. Genet. 17, 284-299. doi: 10.1038/nrg.2016.13

Finer, S., Iqbal, M. S., Lowe, R., Ogunkolade, B. W., Pervin, S., Mathews, C., et al. (2016). Is famine exposure during developmental life in rural Bangladesh associated with a metabolic and epigenetic signature in young adulthood? A historical cohort study. BMJ Open 6:e011768. doi: 10.1136/bmjopen-2016011768 
Fraga, M. F., Ballestar, E., Paz, M. F., Ropero, S., Setien, F., Ballestar, M. L., et al. (2005). Epigenetic differences arise during the lifetime of monozygotic twins. Proc. Natl. Acad. Sci. U.S.A. 102, 10604-10609. doi: 10.1073/pnas.0500398102

Fulop, T., Larbi, A., Dupuis, G., Le Page, A., Frost, E. H., Cohen, A. A., et al. (2018). Immunosenescence and inflamm-aging as two sides of the same coin: Friends or foes? Front. Immunol. 8:1960. doi: 10.3389/fimmu.2017.01960

Garmendia, M. L., Corvalan, C., and Uauy, R. (2014). Assessing the public health impact of developmental origins of health and disease (DOHaD) nutrition interventions. Ann. Nutr. Metab. 64, 226-230. doi: 10.1159/000365024

Godfrey, K. M., Lillycrop, K. A., Burdge, G. C., Gluckman, P. D., and Hanson, M. A. (2007). Epigenetic mechanisms and the mismatch concept of the developmental origins of health and disease. Pediatr. Res. 61, 5R-10R. doi: 10.1203/pdr. 0b013e318045bedb

Gordon, L., Joo, J. H., Andronikos, R., Ollikainen, M., Wallace, E. M., Umstad, M. P., et al. (2011). Expression discordance of monozygotic twins at birth: effect of intrauterine environment and a possible mechanism for fetal programming. Epigenetics 6, 579-592. doi: 10.4161/epi.6.5.15072

Gravina, S., and Vijg, J. (2010). Epigenetic factors in aging and longevity. Pflugers Arch. 459, 247-258. doi: 10.1007/s00424-009-0730-7

Greer, E. L., Becker, B., Latza, C., Antebi, A., and Shi, Y. (2016). Mutation of C. elegans demethylase spr-5 extends transgenerational longevity. Cell Res. 26, 229-238. doi: 10.1038/cr.2015.148

Greer, E. L., Maures, T. J., Hauswirth, A. G., Green, E. M., Leeman, D. S., Maro, G. S., et al. (2010). Members of the H3K4 trimethylation complex regulate lifespan in a germline-dependent manner in C. elegans. Nature 466, 383-387. doi: 10.1038/nature09195

Greer, E. L., Maures, T. J., Ucar, D., Hauswirth, A. G., Mancini, E., Lim, J. P., et al. (2011). Transgenerational epigenetic inheritance of longevity in Caenorhabditis elegans. Nature 479, 365-373. doi: 10.1038/nature10572

Grolleau-Julius, A., Ray, D., and Yung, R. L. (2010). The role of epigenetics in aging and autoimmunity. Clin. Rev. Allergy Immunol. 39, 42-50. doi: 10.1007/s12016009-8169-3

Heijmans, B. T., Tobi, E. W., Stein, A. D., Putter, H., Blauw, G. J., Susser, E. S., et al. (2008). Persistent epigenetic differences associated with prenatal exposure to famine in humans. Proc. Natl Acad. Sci. U.S.A. 105, 17046-17049. doi: 10. 1073/pnas.0806560105

Heo, H. J., Tozour, J. N., Delahaye, F., Zhao, Y., Cui, L., Barzilai, N., et al. (2016). Advanced aging phenotype is revealed by epigenetic modifications in rat liver after in utero malnutrition. Aging Cell 15, 964-972. doi: 10.1111/acel.12505

Herman, J. J., Spencer, H. G., Donohue, K., and Sultan, S. E. (2014). How stable 'should' epigenetic modifications be? Insights from adaptive plasticity and bet hedging. Evolution 68, 632-643. doi: 10.1111/evo.12324

Hochberg, Z., Feil, R., Constancia, M., Fraga, M., Junien, C., Carel, J. C., et al. (2011). Child health, developmental plasticity, and epigenetic programming. Endocr. Rev. 32, 159-224. doi: 10.1210/er.2009-0039

Holliday, R. (1987). The inheritance of epigenetic defects. Science 238, 163-170. doi: $10.1126 /$ science. 3310230

Horvath, S. (2013). DNA methylation age of human tissues and cell types. Genome Biol. 14:R115. doi: 10.1186/gb-2013-14-10-r115

Horvath, S., and Raj, K. (2018). DNA methylation-based biomarkers and the epigenetic clock theory of ageing. Nat. Rev. Genet. 19, 371-384. doi: 10.1038/ s41576-018-0004-3

Iurlaro, M., von Meyenn, F., and Reik, W. (2017). DNA methylation homeostasis in human and mouse development. Curr. Opin. Genet. Dev. 43, 101-109. doi: 10.1016/j.gde.2017.02.003

Jasiulionis, M. G. (2018). Abnormal epigenetic regulation of immune system during aging. Front. Immunol. 9:197. doi: 10.3389/fimmu.2018.00197

Jung, M., and Pfeifer, G. P. (2015). Aging and DNA methylation. BMC Biol. 13:7. doi: 10.1186/s12915-015-0118-4

Kananen, L., Marttila, S., Nevalainen, T., Kummola, L., Junttila, I., Mononen, N., et al. (2016). The trajectory of the blood DNA methylome ageing rate is largely set before adulthood: evidence from two longitudinal studies. Age 38:65. doi: 10.1007/s11357-016-9927-9

Kishimoto, S., Uno, M., Okabe, E., Nono, M., and Nishida, E. (2017). Environmental stresses induce transgenerationally inheritable survival advantages via germline-to-soma communication in Caenorhabditis elegans. Nat. Commun. 8:14031. doi: 10.1038/ncomms14031
Kramer, A., Bekeschus, S., Bröker, B. M., Schleibinger, H., Razavi, B., and Assadian, O. (2013). Maintaining health by balancing microbial exposure and prevention of infection: the hygiene hypothesis versus the hypothesis of early immune challenge. J. Hosp. Infect. 83, S29-S34. doi: 10.1016/S0195-6701(13) 60007-9

Last, J. M. (ed.). (1995). A Dictionary of Epidemiology, 3rd Edn. New York, NY: Oxford University Press.

Levine, M. E., Lu, A. T., Quach, A., Chen, B. H., Assimes, T. L., Bandinelli, S., et al. (2018). An epigenetic biomarker of aging for lifespan and healthspan. Aging 10, 573-591. doi: 10.18632/aging.101414

Li, S., Wong, E. M., Dugué, P. A., McRae, A. F., Kim, E., Joo, J. E., et al. (2018). Genome-wide average DNA methylation is determined in utero. Int. J. Epidemiol. doi: 10.1093/ije/dyy028 [Epub ahead of print].

Li, Y., and Tollefsbol, T. O. (2016). Age-related epigenetic drift and phenotypic plasticity loss: implications in prevention of age-related human diseases. Epigenomics 8, 1637-1651. doi: 10.2217/epi-2016-0078

Lumey, L. H., Stein, A. D., Kahn, H. S., van der Pal-de Bruin, K. M., Blauw, G. J., Zybert, P. A., et al. (2007). Cohort profile: the Dutch Hunger Winter families study. Int. J. Epidemiol. 36, 1196-1204. doi: 10.1093/ije/dym126

Lumey, L. H., Stein, A. D., and Susser, E. (2011). Prenatal famine and adult health. Annu. Rev. Public Health 32, 237-262. doi: 10.1146/annurev-publhealth031210-101230

Lumey, L. H., Terry, M. B., Delgado-Cruzata, L., Liao, Y., Wang, Q., Susser, E., et al. (2012). Adult global DNA methylation in relation to pre-natal nutrition. Int. J. Epidemiol. 41, 116-123. doi: 10.1093/ije/dyr137

Maia Rda, R., and Wünsch Filho, V. (2013). Infection and childhood leukemia: review of evidence. Rev. Saude Publica 47, 1172-1185. doi: 10.1590/S0034-8910. 2013047004753

Marioni, R. E., Suderman, M., Chen, B. H., Horvath, S., Bandinelli, S., Morris, T., et al. (2018). Tracking the epigenetic clock across the human life course: a meta-analysis of longitudinal cohort data. J. Gerontol. A Biol. Sci. Med. Sci. doi: 10.1093/gerona/gly060 [Epub ahead of print].

Martino, D., Loke, Y. J., Gordon, L., Ollikainen, M., Cruickshank, M. N., Saffery, R., et al. (2013). Longitudinal, genome-scale analysis of DNA methylation in twins from birth to 18 months of age reveals rapid epigenetic change in early life and pair-specific effects of discordance. Genome Biol. 14:R42. doi: 10.1186/gb-201314-5-r42

Mendelsohn, A. R., and Larrick, J. W. (2017). Epigenetic drift is a determinant of mammalian lifespan. Rejuvenation Res. 20, 430-436. doi: 10.1089/rej.2017.2024

Merkwirth, C., Jovaisaite, V., Durieux, J., Matilainen, O., Jordan, S. D., Quiros, P. M., et al. (2016). Two conserved histone demethylases regulate mitochondrial stress-induced longevity. Cell 165, 1209-1223. doi: 10.1016/j.cell.2016. 04.012

Roseboom, T. J., Painter, R. C., van Abeelen, A. F., Veenendaal, M. V., and de Rooij, S. R. (2011). Hungry in the womb: What are the consequences? Lessons from the Dutch famine. Maturitas 70, 141-145. doi: 10.1016/j.maturitas.2011. 06.017

Simpkin, A. J., Hemani, G., Suderman, M., Gaunt, T. R., Lyttleton, O., Mcardle, W. L., et al. (2016). Prenatal and early life influences on epigenetic age in children: a study of mother-offspring pairs from two cohort studies. Hum. Mol. Genet. 25, 191-201. doi: 10.1093/hmg/ddv456

Simpkin, A. J., Howe, L. D., Tilling, K., Gaunt, T. R., Lyttleton, O., McArdle, W. L., et al. (2017). The epigenetic clock and physical development during childhood and adolescence: longitudinal analysis from a UK birth cohort. Int. J. Epidemiol. 46, 549-558. doi: 10.1093/ije/dyw307

Souren, N. Y., Lutsik, P., Gasparoni, G., Tierling, S., Gries, J., Riemenschneider, M., et al. (2013). Adult monozygotic twins discordant for intra-uterine growth have indistinguishable genome-wide DNA methylation profiles. Genome Biol. 14:R44. doi: 10.1186/gb-2013-14-5-r44

Strachan, D. P. (1989). Hay fever, hygiene, and household size. BMJ 299, 1259-1260. doi: 10.1136/bmj.299.6710.1259

Tan, Q., Frost, M., Heijmans, B. T., von Bornemann Hjelmborg, J., Tobi, E. W., Christensen, K., et al. (2014). Epigenetic signature of birth weight discordance in adult twins. BMC Genomics 15:1062. doi: 10.1186/1471-2164-15-1062

Tarry-Adkins, J. L., and Ozanne, S. E. (2014). The impact of early nutrition on the ageing trajectory. Proc. Nutr. Soc. 73, 289-301. doi: 10.1017/ S002966511300387X 
Tarry-Adkins, J. L., and Ozanne, S. E. (2017). Nutrition in early life and ageassociated diseases. Ageing Res. Rev. 39, 96-105. doi: 10.1016/j.arr.2016.08.003

Tobi, E. W., Goeman, J. J., Monajemi, R., Gu, H., Putter, H., Zhang, Y., et al. (2014). DNA methylation signatures link prenatal famine exposure to growth and metabolism. Nat. Commun. 5:5592. doi: 10.1038/ncomms6592

Tobi, E. W., Lumey, L. H., Talens, R. P., Kremer, D., Putter, H., Stein, A. D., et al. (2009). DNA methylation differences after exposure to prenatal famine are common and timing- and sex-specific. Hum. Mol. Genet. 18, 4046-4053. doi: $10.1093 / \mathrm{hmg} / \mathrm{ddp} 353$

Tobi, E. W., Slieker, R. C., Luijk, R., Dekkers, K. F., Stein, A. D., Xu, K. M., et al. (2018). DNA methylation as a mediator of the association between prenatal adversity and risk factors for metabolic disease in adulthood. Sci. Adv. 4:eaao4364. doi: 10.1126/sciadv.aao4364

Tobi, E. W., Slieker, R. C., Stein, A. D., Suchiman, H. E., Slagboom, P. E., van Zwet, E. W., et al. (2015). Early gestation as the critical time-window for changes in the prenatal environment to affect the adult human blood methylome. Int. J. Epidemiol. 44, 1211-1223. doi: 10.1093/ije/dyv043

Tsai, P. C., Van Dongen, J., Tan, Q., Willemsen, G., Christiansen, L., Boomsma, D. I., et al. (2015). DNA Methylation changes in the IGF1R gene in birth weight discordant adult monozygotic twins. Twin Res. Hum. Genet. 18, 635-646. doi: 10.1017/thg.2015.76

Vaiserman, A. (2015). Epidemiologic evidence for association between adverse environmental exposures in early life and epigenetic variation: a potential link to disease susceptibility? Clin. Epigenetics 7:96. doi: 10.1186/s13148-0150130-0

Vaiserman, A., Koliada, A., and Lushchak, O. (2018). Developmental programming of aging trajectory. Ageing Res. Rev. 47, 105-122. doi: 10.1016/j.arr.2018. 07.007

Vaiserman, A. M. (2008). Epigenetic engineering and its possible role in anti-aging intervention. Rejuvenation Res. 11, 39-42. doi: 10.1089/rej.2007.0579
Vaiserman, A. M. (2010). Hormesis, adaptive epigenetic reorganization, and implications for human health and longevity. Dose Response 8, 16-21. doi: 10.2203/dose-response.09-014.Vaiserman

Vaiserman, A. M. (2014). Early-life nutritional programming of longevity. J. Dev. Orig. Health Dis. 5, 325-338. doi: 10.1017/S2040174414000294

van Abeelen, A. F., Veenendaal, M. V., Painter, R. C., de Rooij, S. R., Dijkgraaf, M. G., Bossuyt, P. M., et al. (2012). Survival effects of prenatal famine exposure. Am. J. Clin. Nutr. 95, 179-183. doi: 10.3945/ajcn.111.022038

Xia, B., and de Belle, J. S. (2016). Transgenerational programming of longevity and reproduction by post-eclosion dietary manipulation in Drosophila. Aging 8, 1115-1134. doi: 10.18632/aging.100932

Xia, B., Gerstin, E., Schones, D. E., Huang, W., and Steven de Belle, J. (2016). Transgenerational programming of longevity through $\mathrm{E}(\mathrm{z})$-mediated histone H3K27 trimethylation in Drosophila. Aging 8, 2988-3008. doi: 10.18632/aging. 101107

Yashin, A. I., Ukraintseva, S. V., De Benedictis, G., Anisimov, V. N., Butov, A. A., Arbeev, K., et al. (2001). Have the oldest old adults ever been frail in the past? A hypothesis that explains modern trends in survival. J. Gerontol. A. Biol. Sci. Med. Sci. 56, B432-B442. doi: 10.1093/gerona/56.10.B432

Conflict of Interest Statement: The author declares that the research was conducted in the absence of any commercial or financial relationships that could be construed as a potential conflict of interest.

Copyright (C) 2018 Vaiserman. This is an open-access article distributed under the terms of the Creative Commons Attribution License (CC BY). The use, distribution or reproduction in other forums is permitted, provided the original author(s) and the copyright owner(s) are credited and that the original publication in this journal is cited, in accordance with accepted academic practice. No use, distribution or reproduction is permitted which does not comply with these terms. 